\title{
TAGUNG
}

\section{Deutschland in der Europäischen Union - Eine Standortbestimmung}

\author{
Tobias Gierling und Tanja Leppik*
}

Die Frage nach der Rolle Deutschlands in der Europäischen Union ist ein zeitloses Thema der deutschen und internationalen Politikwissenschaft. Die bedeutsame Rolle Deutschlands für Richtung und Dynamik des europäischen Integrationsprozesses wird nicht nur auf die geografische Lage Deutschlands in der Mitte Europas zurück geführt, sondern auch auf seine spezifischen politischen, ökonomischen, demografischen und historischen Entwicklungsaspekte. Doch hängt Europas $\mathrm{Zu}$ kunft von der Zukunft Deutschlands ab, wie es kürzlich der polnische Präsident Kwasniewski äußerte? Diese Frage wurde im Rahmen der Jahreskonferenz des Arbeitskreises Europäische Integration aus unterschiedlichen Perspektiven beleuchtet und interdisziplinär diskutiert. Peter-Christian Müller-Graff eröffnete die Tagung und verband die grundsätzliche Frage nach der Rolle Deutschlands in der Europäischen Union mit der aktuellen Krise der Union nach den gescheiterten Verfassungsreferenden.

\section{Kontinuität oder Wandel der Europapolitik?}

Zunächst analysierte Andreas Maurer die Strukturen der deutschen Europapolitik und erörterte die Frage, ob es eine neue deutsche Europapolitik gebe. Um die deutsche Europapolitik ,vermessen ‘ zu können, griff er auf ein Bewertungsraster der drei I's - Interessen, Institutionen und Ideen - zurück.

Das Grundmuster der Europapolitik in Deutschland wird nach Maurer von vier Faktoren beeinflusst: Exogene Faktoren, wie die

\section{Deutschlands Rolle in der Europäischen Union}

Jahreskolloquium des Arbeitskreises Europäische Integration

Berlin, 17./18. November 2005

\section{Einführung in das Thema}

Prof. Dr. Dr. h. c. Peter-Christian MÜLLER-

GRAFF, Vorsitzender des Vorstands des Arbeitskreises Europäische Integration, Universität Heidelberg

\section{Grundfragen}

Nach der Referendenzäsur: Neue Deutsche Europapolitik in und nach der Denkpause über den Verfassungsvertrag?

Dr. Andreas MAURER, Stiftung Wissenschaft und Politik, Berlin

Kommentar: Prof. Dr. Michael KREILE, Humboldt-Universität Berlin

Nach der Referendenzäsur: Neue Erwartungen anderer Staaten an die Rolle Deutschlands in Europa?

Mateusz FALKOWSKI, Institute for Public Affairs, Warschau

Die wirtschaftlichen Perspektiven: Eine vergleichende Analyse für Deutschland und Österreich innerhalb der erweiterten und harmonisierenden Europäischen Union und in der Weltwirtschaft

Prof. Dr. Fritz BREUSS, Wirtschaftsuniversität Wien

Verfassungsvertragliche Perspektiven und Grundgesetz: Offenheit, Bedingungen und Grenzen der Beteiligung Deutschlands an Neuerungen der Europäischen Union

Prof. Dr. Ulrich FASTENRATH, Technische Universität Dresden

* Tobias Gierling, Praktikant in der Forschungsgruppe EU-Integration, Stiftung für Wissenschaft und Politik, Berlin.

Tanja Leppik, Netzwerk Europäische Bewegung Deutschland, Berlin. 
Globalisierung; EU-außen induzierte Faktoren, beispielsweise Reformen des Rates; binnen-induzierte Faktoren, wie das nationale System der europapolitischen Koordinierung, und endogene Faktoren, beispielsweise die Grundstimmungen in der Bevölkerung. Diese Faktoren veränderten die Union beziehungsweise Deutschland, haben aber auch Rückwirkungen auf die nationalstaatlichen Entscheidungsmuster in der Europapolitik. Derzeit gebe es in der deutschen Europapolitik einige sich andeutende Veränderungen. Die deutsche Europapolitik sei heute stärker durchsetzt von einem Kosten-Nutzen-Denken und die entscheidenden Akteure achteten bewusster und häufiger auf ihre mediale Wirkung. So sei es das vorrangige Ziel der Bundesregierung in den Verhandlungen zum Vertrag über eine Verfassung für Europa (VVE) gewesen, die Rolle Deutschlands in der Europäischen Union zu stärken. Dies habe sich etwa in dem Bestreben gezeigt, den Europäischen Rat sichtbar aufzuwerten und das Prinzip der doppelten Mehrheit für Mehrheitsvoten im Ministerrat einzuführen. Insgesamt sei der Eindruck entstanden, dass Deutschland, Frankreich und Großbritannien versuchten, die Kommission faktisch zu instrumentalisieren und als Instrument des Europäischen Rates zu verwenden. In der derzeitigen Denkpause sei ein allgemeines ,Stillhalten' sowie Kritik an jeglichen Vorschlägen zu einer teilweisen Umsetzung des VVE zu bemerken, obschon die Umsetzung einzelner Elemente des VVE durchaus möglich wäre.

Maurer formulierte abschließend einige Vorschläge für die künftige deutsche Europapolitik: Es müsse mehr deutsche Initiativen mit anderen EU-Partnern wie beispielsweise Rom oder Madrid geben. Deutschland müsse seiner - auch historischen - Verantwortung gerecht werden und rechtsextremistischen und -populistischen Tendenzen in der erweiterten Union entgegenwirken sowie das gemeinsame europäische Wertefundament festigen. Außerdem sei es wichtig, über mögliche Alternativen zur Vollmitgliedschaft nachzudenken.

\section{Einzelfragen}

Deutschland in der Finanzverfassung der EU: Steigende Netto-Belastung des Wachstumsschlusslichts?

Dr. Friedrich Heinemann, Universität Mannheim

Perspektiven des demographischen Profils von Deutschland im Vergleich: Auswirkungen auf die Europäische Union

Prof. Dr. Josef Schmid, Universität Bamberg

Perspektiven der deutschen Sprache in der Europäischen Union

Dr. Katharina von Ruckteschell-Katte, Goethe-Institut e. V., München

Import und Export von Rechtsdenken und Rechtspraxis: Die deutsche Rechtsordnung innerhalb der Europäischen Union

PD Dr. Christoph Teichmann, Universität Heidelberg

PD Dr. Hans-Peter Folz, Universität Augsburg/Universität der Bundeswehr München

Die Rolle von Bundestag und Bundesrat in der europäischen Rechtsetzung

Dr. Cordula Janowski, Zentrum für Europäische Integrationsforschung, Bonn

Deutsche Föderalismuserfahrungen als Orientierungspunkte für die Zukunft der Europäischen Union?

Prof. Dr. Rudolf Hrbek, Präsident des Arbeitskreises Europäische Integration, Universität Tübingen

Michael Kreile stimmte den Aussagen Maurers größtenteils zu. Allerdings sei Deutschland seiner Meinung nach gerade nicht dazu berufen, gegenüber anderen Staaten als Hüter der moralischen Werte aufzutreten. Hinsichtlich der deutschen Europapolitik stellte er eine große Kontinuität fest, deshalb seien derzeit keine neuen großen Projekte notwendig. Allerdings habe die Ratifikationskrise gezeigt, dass die Innenpolitik der Mitgliedstaaten vielfach unvorhersehbarer sei, als vielfach angenommen. Deshalb gebe es vor allem einen Konsolidierungsbedarf, etwa bei der finanziellen und institutionellen Absicherung der Erweiterung, aber auch bei den nationalen Haushalten. Außerdem gebe es viele gute Gründe - wie etwa die Angst vor der Globali- 
sierung, die steigende Arbeitslosigkeit - die Enttäuschungen auszuloten und den Handlungsbedarf auf Grundlage der Innenpolitik zu definieren.

Die Erwartungen der anderen Mitgliedstaaten an die deutsche Europapolitik wurden von Mateusz Falkowski vorgestellt. $\mathrm{Er}$ beschränkte sich dabei auf die Erwartungen Polens und befasste sich mit der Frage, ob die polnischen Erwartungen an Deutschland neu und speziell an Deutschland gerichtet seien. In Polen zeigten demoskopische Umfragen, dass fast die Hälfte der polnischen Bevölkerung denke, dass Polen über keinen echten Verbündeten in der Europäischen Union verfüge und lediglich durch die USA unterstützt werde. Ursache dieser polnischen Skepsis sei die weit verbreitete Angst der Polen vor dem großen Nachbarn Russland. Zudem seien über 50 Prozent der Bevölkerung in Folge der Debatten über den VVE davon überzeugt, dass Frankreich und Deutschland eine Abneigung gegenüber Polen hätten. Derzeit gebe es zwar eine große Unterstützung für die Mitgliedschaft in der Europäischen Union, gerade auch bei den Bevölkerungsgruppen, die nunmehr von EU-Fördergeldern profitierten. Eine weitere Vertiefung des Integrationsprozesses, etwa durch den VVE, werde hingegen nicht unterstützt. Insgesamt existierten eigentlich keine neuen, sondern vielmehr die alten $\mathrm{Er}$ wartungen Polens an Deutschland und Europa als Sicherheitsgaranten im Hinblick auf Russland.

Von der Europäischen Union erwarte Polen vor allem eine aktive Politik im Osten. Die Erwartungen an Deutschland seien besonders struktureller und institutioneller, aber nicht inhaltlicher Natur. Der vorrangige Wunsch sei es, beim Finanzrahmen und in der Ostpolitik als gleichberechtigter Partner eingebunden zu werden. In der Europäischen Union solle keine Gruppenbildung betrieben werden, die $\mathrm{zu}$ einer Zwei-Klassen-Gesellschaft führen könne, deshalb solle Deutschland nicht alleine mit Moskau und Paris sprechen. Falkowski wies darauf hin, dass es allerdings eine große
Skepsis in Polen gebe, ob derzeit überhaupt etwas von Deutschland erwartet werden könne. Zentrale Punkte für alle Parteien seien die Ostpolitik, die deutsche Unterstützung im Finanzbereich, die Beibehaltung der deutschen Haushaltszahlungen, die Beendigung der Freizügigkeitsbeschränkung für polnische Arbeitnehmer sowie die Öffnung der Dienstleistungsmärkte. Die Erwartungen zusammenfassend stellte Falkowski fest, dass aus Sicht der polnischen Bevölkerung eine „gute“ Europapolitik nur mit einer starken transatlantischen Beziehung vorstellbar sei, die polnischen Beziehungen mit den USA und mit der Union würden nicht als Gegensatz betrachtet.

Doch in der Europäischen Union gibt es nicht nur unterschiedliche nationale Interessen, auch innerhalb eines Staates gibt es verschiedene Akteure mit eigenen Anliegen. So ist beispielsweise zu fragen, welchen Einfluss nationale Parlamente auf die Entstehung von Europapolitik ausüben können. Cordula Janowski befasste sich in ihrem Vortrag mit den spezifischen Mitwirkungsrechten von Bundestag und Bundesrat. Sie wies darauf hin, dass es seit dem Vertrag von Maastricht und dem Maastricht-Urteil des Bundesverfassungsgerichts (BVerfG) ein gewandeltes Verständnis der Europapolitik sowie eine verstärkte Diskussion um das Demokratiedefizit und die Entparlamentarisierung der Europäischen Union gebe. In seinem Maastricht-Urteil habe das BVerfG eine duale Legitimation der Europapolitik festgestellt. Europapolitik werde zum einen über das Europäische Parlament, zum anderen über die nationalen Parlamente legitimiert. Dadurch seien die nationalen Parlamente als neuer Akteur ins Spiel gebracht worden. In diesem Zusammenhang sei auch der neue Artikel 23 ins Grundgesetz (GG) eingefügt worden, der die Mitwirkung von Bundestag und Bundesrat regelt und den alten Artikel 24 ersetzt hat.

Janowski führte empirische Daten auf, die belegen, dass angesichts der in den letzten zwanzig Jahren feststellbaren Dynamik der europäischen Rechtsetzung ein Kompetenzverlust der 
nationalen Parlamente feststellbar sei. Deshalb habe der Bundestag befürchtet, zur reinen Registriermaschine zu werden und mehr Kompetenzen in der Europapolitik gefordert. Allerdings habe sich der Bundestag im Gegensatz zum Bundesrat vergleichsweise spät um Mitwirkung an der deutschen Europapolitik bemüht. Zudem zeige ein Vergleich von Verfassungsnorm und Verfassungspraxis, dass der Bundestag zwar über einen Europa-Ausschuss mit Verfassungsrang und diversen Sonderrechten verfüge, dass dieser Ausschuss seine Rechte in der Praxis aber relativ selten nutze. Außerdem bearbeite er vorrangig Grundsatzangelegenheiten, während fachliche Dokumente federführend von anderen Ausschüssen behandelt würden. Wichtig ist nach Janowski, gerade angesichts der Vielzahl der EU-Vorlagen, die Selektion der Dokumente sowie die zeitliche Koordinierung der Behandlung im Parlament. Der VVE biete in diesem Zusammenhang neue Möglichkeiten zur Mitwirkung. Denn durch den vorgesehenen Frühwarnmechanismus würde die direkte und frühzeitige Zuleitung der Dokumente durch die Kommission an die nationalen Parlamente sichergestellt.

Mit Bezug auf die Hauptmerkmale des Föderalismus zeigte Rudolf Hrbek, dass die Europäische Union als System mit föderalem Charakter begriffen werden könne. Mit den Mitgliedstaaten und der Europäischen Gemeinschaft seien auch hier zwei Ebenen vorhanden. Es existieren sowohl geteilte wie auch konkurrierende Kompetenzen zwischen den Ebenen, die eine direkte Wirkung für die Bürger in einem solchen Herrschaftsverband entfalten. Die Europäische Union sei bei der Rechtssetzung mitverantwortlich, wobei sie sich seit Maastricht auf die Prinzipien der Subsidiarität und Proportionalität beziehe. Während die Bereiche, in denen einstimmig im Ministerrat entschieden werde, eher der Konstruktion eines Staatenbundes ähnelten, tendiere die Europäische Union durch zunehmende qualifizierte Mehrheitsentscheidungen in Richtung eines Bundesstaates. Mit dem Europäischen Gerichtshof (EuGH) sei zudem eine richterliche Instanz vorhanden, die im Konfliktfall zwi- schen Staats- und Gemeinschaftsebene entscheide. Typologisch entspreche die Europäische Union nicht einem dualen, sondern eher einem kooperativen Föderalismus.

Durch den VVE werde die föderale Qualität der Europäischen Union erhöht. Hrbek erwähnte hier das Prinzip der Unionstreue, nach dem sich die Union und die Mitgliedstaaten gegenseitig unterstützten; dass die Devise der Europäischen Union, in Vielfalt geeint zu sein, ausdrücklich betont werde; die explizite Anerkennung der lokalen und regionalen Ebene, die den Mehrebenencharakter zum Ausdruck bringe; eine neue Kompetenzordnung; die Ausweitung der Rechte für das Europäisches Parlament und die Zunahme qualifizierter Mehrheitsentscheidungen.

Anschließend blickte Hrbek auf die deutschen Erfahrungen mit dem Föderalismus und die sich daraus potentiell ergebenden Orientierungspunkte für die Europäische Union. Unter Rückbezug auf Falkowski und die polnischen Bedenken gegenüber Gruppenbildungen in der Europäischen Union verwies er auf die bi- und multilateralen Verträge zwischen den deutschen Bundesländern, die Kooperationsmuster darstellen würden, die auf die Europäische Union angewandt für eine Gruppenbildung in kleineren Arrangements sprechen könne. Dies sei schlichtweg funktional, was bedeute, dass Polen solche Gruppenbildungen nicht stets als gegen sich selbst gerichtet betrachten solle. Im Fazit und Ausblick stellte Hrbek die aus seiner Sicht rhetorische Frage, ob der europäische Integrationsprozess, wie er sich in seiner Dynamik darstelle, nicht geradezu Verflechtung brauche. Aus deutscher Perspektive sei klar, dass es Verflechtung geben müsse, ohne dass es zu den in Deutschland häufig kritisierten Mängeln an der Politikverflechtung und starken Unitarisierungstendenzen komme.

Wechselverhältnis zwischen deutschem und europäischem Recht

Hans-Peter Folz stellte die gegenseitige Beeinflussung des Rechtsdenkens zwischen eu- 
ropäischer und deutscher Ebene beim Grundrechtsschutz ins Zentrum seines Vortrags. Über den Dualismus von supranationaler Rechtssetzung und deutschem Verfassungsrecht hinaus, müsse aufgrund der besonderen Beziehung zur Europäischen Menschenrechtskonvention (EMRK) von einem Dreiecksverhältnis zwischen Luxemburg, Karlsruhe und Straßburg und deren Wechselbeziehungen gesprochen werden. Der Anstoß zur Entwicklung des Grundrechtsschutzes im Gemeinschaftsrecht ginge maßgeblich vom BVerfG mit seiner SolangeRechtsprechung aus. Der EuGH entwickelte einen Grundrechtsschutz durch ungeschriebene Allgemeine Rechtsgrundsätze, die er aus den gemeinsamen Verfassungstraditionen der Mitgliedstaaten ableitete. Zudem ziehe der EuGH die EMRK als Rechtserkenntnisquelle heran, wodurch der EuGH ein erhebliches $\mathrm{Maß}$ an Flexibilität im Rahmen der Herausbildung eines genuin gemeinschaftsrechtlichen Grundrechtsschutzes erhielt. Der Art. 6 Abs. II EUV verdeutliche, dass die Rechtsfortbildung des EuGH die Anerkennung der Mitgliedstaaten fand. Sollte der VVE in Kraft treten würde eine Bindung des Grundrechtschutzes an die EMRK gemäß Art. II-112 Abs. 3 VVE fortbestehen. Der Rechtsprechung des EuGH sowie des Straßburger Gerichtshofes seien hier, zur Vermeidung von Konventionsverletzungen, keinerlei Abweichungen erlaubt. $\mathrm{Zu}$ berücksichtigen sei in diesem Zusammenhang der Art. I-9 Abs. 2 VVE, der mittelfristig einen Beitritt der Europäischen Union zur EMRK vorsehe. Die Europäische Union unterliege jedoch bereits einer indirekten Kontrolle durch den Europäischen Gerichtshof für Menschenrechte.

Als unmittelbarer Prüfungsmaßstab gelte die EMRK, mangels Verfassungsrangs, vor dem BVerfG nicht, so dass eine Verfassungsbeschwerde nicht unmittelbar auf eine Verletzung der EMRK gestützt werden könne. Trotzdem bestünde hier das Gebot der konventionskonformen Auslegung des einfachen Bundesrechts wie des deutschen Verfassungsrechts. Eine Pflicht zur Berücksichtigung der
EMRK bei der Entscheidungsfindung gelte für alle Behörden und Gerichte der Bundesrepublik. Einige Fälle zeigten, dass eine stärkere Auseinandersetzung mit der EMRK zu empfehlen sei, dennoch würden die Grundsätze des BVerfG eine hinreichende Berücksichtigung der EMRK erlauben.

Nach der Darstellung verschiedener Konzepte zur Auslegung von Grundrechten, zur Rechtfertigung von Grundrechtseingriffen und zur Schutzpflichtdogmatik, sei insgesamt festzuhalten, dass die Auseinandersetzung auf dem Gebiet des Grundrechtsschutzes deutlich von gegenseitiger Einflussnahme geprägt sei, die parallele Entwicklungen, aber auch Divergenzen aufweise.

Ulrich Fastenrath befasste sich mit den europabezogenen Regelungen des GG und den verfassungsvertraglichen Perspektiven. Seine Ausgangsthese lautete mit Bezug auf Art. 23 Abs. 1 GG, dass die darin festgeschriebene Verpflichtung Deutschlands, bei der Entwicklung der Europäischen Union die Demokratie, Rechtsstaatlichkeit, Sozialstaatlichkeit, den föderativen Aufbau und die Subsidiarität zu gewährleisten, bisher geglückt sei. Mit Blick auf die Zukunft ginge es daher in seinen folgenden Ausführungen um zwei wesentliche Aspekte: erstens den Schutz der deutschen Staatlichkeit und zweitens um die Anforderungen zur Sicherung der Demokratie.

Anhand der Drei-Elemente-Lehre von Jellinek, die den Staat durch die Elemente Staatsgebiet, Staatsvolk und Staatsherrschaft definiert, verdeutlichte er, dass die Staatlichkeit der Union unter Betrachtung der derzeitigen Rechtslage hinsichtlich aller drei Elemente bezweifelt werden könne. Bei der Betrachtung des VVE sei dies insofern problematischer, da dieser einen doppelten Ableitungszusammenhang festschreibe, wonach nicht mehr nur die Staaten als Herren der Verträge anzusehen seien, sondern explizit auf den Willen der Bürger Bezug genommen werde. Die Analyse des VVE könne Zweifel an der Bewahrung der Souveränität der Mitglied- 
staaten aufkommen lassen. Ein absoluter Vorrang der EU-Regelungen sei aber in Bezug auf den Fall Costa/ENEL und die Solange IRechtssprechung des BVerfG zu bezweifeln. Durch letzteres Urteil bestehe eine Schranke, nach der die Hoheitsgewalt nicht so weit übertragen werden kann, dass sie die Grundstruktur des GG verletzte, die besonders in Art. 1 und 20 GG zu sehen ist.

Fastenrath erörterte die Frage inwieweit ein Schutz der deutschen Staatlichkeit durch die Art. 23, 79 und 146 GG bestünde. Bezogen auf die Sicherung der Demokratie dürfe laut des Maastricht-Urteils die Europäische Integration nur soweit gehen, solange noch ein demokratisches Zusammenleben möglich sei. Ein wesentlicher Schritt zur Behebung des Demokratiedefizits sei durch eine weitgehende Kompetenzverlagerung vom Ministerrat zum Europäischen Parlament und einer besseren Kontrolle des Ministerrates möglich. Um Richtungswahlen auf europäischer Ebene zu ermöglichen müssten die europäischen Parteienbünde mit Spitzenkandidaten antreten, womit ein wesentlicher Schritt zur Demokratisierung getan werden könne. Neben institutionellen Änderungen seien mit Blick auf die Bürger allerdings auch inhaltliche Korrekturen notwendig, die sich vor allem mit der Schaffung klarer Kompetenzbereiche befassen sollten, wobei dies gerade für kleine und mittlere Unternehmen im Bereich des europäischen Wirtschafts- und Handelsrechts bedeutsam sei.

Christoph Teichmann konzentrierte sich auf die Frage des Im- und Exports deutschen Rechtsdenkens im Bereich des Unternehmensrechts. $\mathrm{Zu}$ den Wesensmerkmalen des deutschen Unternehmensrechts gehörten folgende Aspekte: die Zweispurigkeit der Kapitalgesellschaften von der AG und der $\mathrm{GmbH}$, Gläubigerschutz durch Mindestkapital, die Trennung von Vorstand und Aufsichtsrat und die Mitbestimmung der Arbeitnehmer. In Deutschland existierte zunächst die AG, die ihren Boom sowie die erste Krise zu Beginn der 1870er Jahre erlebte. Die GmbH sei erst
1892 eingeführt worden und galt als, AG des kleinen Mannes'. Heutzutage sei sie in Deutschland die weitaus verbreitetere Kapitalgesellschaftsform. Im Gegensatz zum deutschen Recht existiere nach britischem Recht die ,company“ als einzige Rechtsform, allerdings bestehe mit der „Limited“ hier eine spezielle Unterkategorie.

Der Einfluss des deutschen Rechtsdenkens habe sich zum einen hinsichtlich des Gläubigerschutzes durch Mindestkapital in der zweiten Richtlinie zur AG wie auch durch die Einführung der $\mathrm{GmbH}$ in Dänemark und den Niederlanden gezeigt. Die Zweispurigkeit wurde somit exportiert, wogegen sich Großbritannien wehrte, das darauf bedacht gewesen sei, die einheitliche Rechtsform zu wahren. Die duale Konstruktion von Vorstand und Aufsichtsrat sowie der Grundsatz der personellen Trennung sei Teichmann zufolge eine deutsche Idee. Einfluss übte sie auf die Strukturrichtlinie der Europäischen Aktiengesellschaft aus. Das gleiche gelte für die Mitbestimmung der Arbeitnehmer. Teichmann befasste sich anschließend mit dem Vordringen konkurrierender Regelungsmodelle, die sich im Bereich des Bilanzrechts, dem Selbstschutz der Gläubiger sowie der Debatte um ,Corporate Governance ' fänden. So würden die Bilanzen in Deutschland nicht nach dem internationalen ,true and fair view“-Prinzip, sondern nach dem deutschen ,Vorsichtsprinzip“ erstellt. Die ,Corporate Governance'-Debatte stelle die Effizienz in den Mittelpunkt. Im Zuge der Debatte gerate das Vorstand/ Aufsichtsrat-Modell, ebenso wie die Mitbestimmung, die als Fremdkörper betrachtet werde, in die Defensive. Der Ansatz, nach dem das europäische Gesellschaftsrecht harmonisiert werden solle, befinde sich momentan in einer Blockadesituation auf Grund der Existenz konkurrierender Modelle. Zu fragen wäre hier demnach, ob es zu einer Konvergenz des monistischen und des dualistischen Leitungsmodells kommt, oder ob Unternehmen es sich selbst aussuchen können sollten. Die Verschmelzung in eine andere Rechtsform sei jedenfalls bisher nicht möglich. 
Beim Thema der Mitbestimmung könne mit Hinweis auf die Verringerung der Informationsasymmetrie durchaus auf deren ökonomischen Nutzen verwiesen werden.

\section{Die wirtschaftliche und finanzielle Situation}

Fritz Breuss befasste sich in seinem Vortrag mit der makroökonomischen Stellung Deutschlands und Österreichs im europäischen und weltwirtschaftlichen Vergleich. Bei der Wohlstandsentwicklung liegen derzeit beide Länder deutlich hinter den USA; beim Körperschaftssteuersatz finden sich beide Länder im Mittelfeld der EU-25 ein. Die Betrachtung der Unternehmenssteuern insgesamt zeige für den Zeitraum 1995 bis 2000, dass diese in allen Ländern der erweiterten Europäischen Union drastisch zurückgegangen seien.

Wesentliche Unterschiede bestünden bei den Arbeitslosenzahlen: In Österreich liege die Arbeitslosenquote ungefähr halb so hoch wie in Deutschland. Zudem falle Deutschland bei der Erfüllung des Stabilitäts- und Wachstumspaktes eindeutig hinter Österreich zurück, da Deutschland bereits im fünften Jahr hintereinander die Kriterien des Paktes durch ein zu hohes Defizit verletze. Deutschland nehme hingegen eine weltweite Spitzenposition im Imund Exportbereich von Gütern und kommerziellen Dienstleistungen sowie bei der Anzahl der Triadepatente ein und weise im Gegensatz zu Österreich eine positive Handelsbilanz auf. Die These von Werner Sinn, die deutsche Wirtschaft laufe Gefahr, sich zu einer, Basarökonomie` zu entwickeln, bezweifelte Breuss. Deutschland sei schon immer eine erfolgreiche ,Händlernation' gewesen. Internationale Vergleiche zur Größe des Dienstleistungssektors sowie der prozentuale Anteil der Industrie an der Gesamtwertschöpfung verdeutlichten dies.

Dennoch falle das Wirtschaftswachstum Deutschlands seit der deutschen Einheit geringer aus als in Österreich. Bei einem Vergleich Ostdeutschlands mit den anderen Transformationsländern lasse sich von einem besonderen Transferschock sprechen, da die
Wachstumsdaten verdeutlichten, dass trotz der hohen Transferzahlungen das Wirtschaftswachstum seit Mitte der 1990er Jahre deutlich geringer ausfalle, als in den neuen EU-Mitgliedstaaten aus Mittel- und Osteuropa.

Dass Österreich im Vergleich „happier“ sei als Deutschland, zeige die sogenannte Glückskurve, die den Prozentsatz der glücklichen und zufriedenen Menschen und das ProKopf-Einkommen erfasse. Zusammenfassend könne festgehalten werden, dass Deutschland noch immer die führende Wirtschaftsmacht in der Europäischen Union sei, die aktuellen Entwicklungen beim Wachstum sowie dem Arbeitsmarkt allerdings suboptimal seien. Im Vergleich dazu sei Österreich als typischer Kleinstaat derzeit flexibler und profitiere stärker von der Ostöffnung und der EU-Erweiterung 2004.

Eine unverzichtbare Voraussetzung jeglicher Politik sind die finanziellen Ressourcen. Für die Europäische Union wird das zur Verfügung stehende Ausgabenvolumen und die Struktur des EU-Haushalts in der Finanziellen Vorausschau festgeschrieben, die für die Jahre 2007 bis 2013 derzeit verhandelt wird. Zentraler Maßstab der Mitgliedstaaten in diesen Verhandlungen ist die nationale Bilanz der Nettozahlungen. Seinen Vortrag über die Finanzverhandlungen begann Friedrich Heinemann allerdings mit der normativen Frage nach dem mit EU-Geldern zu erzielenden „Europäischen Mehrwert". Obwohl diese Frage eigentlich im Vordergrund stehen sollte, werde sie in der Verhandlungspraxis nur unzureichend beachtet und von der Nettosaldo-Betrachtung überlagert. Nach Heinemann ließe sich letzteres zwar rechtfertigen, da der EU-Haushalt transferlastig sei, dennoch sei es seiner Meinung nach im Grundsatz falsch. Er betonte aber auch deutlich die Notwendigkeit einer strengen EU-Haushaltsdisziplin, da ansonsten der Bundeshaushalt signifikant durch die weiteren Bruttobeiträge an den EU-Haushalt belastet werde. Eine Analyse des Kommissionsvorschlags zur Finanziellen Vorausschau zeige, dass die Nettobelastung Deutschlands stark steigen würde. 
Heinemann konstatierte einen Zusammenhang zwischen der Stimmenverteilung im Ministerrat und der Verteilung von EU-Geldern auf einzelne Politikfelder. Zur Berechnung der Nettosalden für spezifische Politikbereiche habe das Zentrum für Europäische Wirtschaftsforschung ein Computerprogramm entwickelt, das auch die Stimmrechte im Rat einbeziehe. Insgesamt werde die Begrenzung des EU-Budgets auf ein Prozent des EU-Bruttonationaleinkommens von einer signifikanten Mehrheit im Rat gestützt. Die Mehrheit der Länder stellten somit potenzielle Verbündete des Nettozahlers Deutschland bei der Einprozent-Forderung dar. Hinsichtlich des Britenrabatts zeigten die Berechnungen, dass bei der Abschaffung, aber auch bei der Kappung des Rabatts, alle Länder bis auf Großbritannien selbst profitieren würden. Heinemann untersuchte zudem die Verteilungswirkungen möglicher Politikfeldumschichtungen. Dabei betrachtete er die politikfeldbezogenen Präferenzen der Mitgliedstaaten am Beispiel der Politikfelder Forschung, Kohäsion und Landwirtschaft unter dem Nettosaldenaspekt. Dabei zeigte sich, dass im Bereich der Landwirtschaftspolitik die Verteilung der gewichteten Stimmen im Rat eher für ein Festhalten am Status quo spreche und Umschichtungen entsprechend schwierig zu erreichen seien.

Dennoch kam Heinemann in seinem Fazit zu der Feststellung, dass eine Absenkung des Budgetvolumens gegenüber dem Kommissionsvorschlag für die Mehrheit der EU-Mitgliedstaaten attraktiv sei und der deutsche Widerstand aufgrund der hohen deutschen Nettozahlungen durchaus glaubwürdig sei. Hinsichtlich des Britenrabattsystems erscheine eine Kappung als einzig realistische Option und Kürzungen der Agrarausgaben erschienen zwar wünschenswert, aber leider nicht realistisch.

Entwicklungen der deutschen Sprache und Altersstruktur

Katharina von Ruckteschell-Katte beschäftigte sich mit den Perspektiven der deutschen Sprache in der Europäischen Union. Sie be- tonte, dass die wichtigste Frage in diesem $\mathrm{Zu}$ sammenhang eigentlich sei, ob die Mehrsprachigkeit in der Europäischen Union eine Zukunft habe. Unter der Prämisse, dass in der Union mehrere Arbeitsprachen beibehalten würden, sei es sehr wahrscheinlich, dass auch das Deutsche zu diesen Sprachen zählen werde. Anschließend erläuterte sie, dass der Wert einer Sprache von der Zahl der Sprecher, der geografischen Verbreitung, dem kulturellen Image sowie vom Ausmaß der medialen Verwendung abhänge. Eine Sprache habe nach von Ruckteschell-Katte zwei Grundfunktionen: sie fungiere als Kommunikationsmittel sowie als Träger und Vermittler soziokultureller Werte. Die Förderung der deutschen Sprache als Träger kultureller Werte sei eine wichtige Aufgabe des GoetheInstituts, für die Haushaltsmittel durch das Auswärtige Amt bereitgestellt würden.

Hinsichtlich der Mehrsprachigkeit in der Europäischen Union hielt sie fest, dass diese in der Europäischen Union fest verankert sei, da sie eine politische Notwendigkeit darstelle. Allerdings hänge die Position der deutschen Sprache auch von der deutschen Politik ab. In Deutschland scheine es mit der Förderung der Mehrsprachigkeit offensichtlich Schwierigkeiten zu geben. So gäbe es erst seit relativ kurzer Zeit in Grundschulen Sprachunterricht, während die Verpflichtung zum Erwerb einer zweiten Fremdsprache selten bestünde. Nach von Ruckteschell-Katte liegt der Ursprung des fehlenden Sprachpatriotismus in Deutschland in der deutschen Geschichte begründet. Allerdings sei das Bild des Deutschen im Ausland sehr viel positiver als hierzulande angenommen. Abschließend erläuterte sie, warum das Deutsche eine positive Perspektive in Europa habe. So fungiere das Deutsche als eine Brückensprache zwischen West und Ost, und die Sprache und Kultur Deutschlands seien erfolgreiche, Exportgüter'. Zudem sei Deutsch inzwischen die am meisten gesprochene Sprache in der Europäischen Union. Von Ruckteschell-Katte betonte aber, dass die Aufgabe weiterhin wichtig bliebe, sich konti- 
nuierlich für die deutsche Sprache in der Union einzusetzen.

Mit der Altersstruktur in Deutschland befasste sich Josef Schmid. Er analysierte das demografische Profil anhand dreier Faktoren: der Geburtenrate, den Sterbefällen und den Wanderungsbewegungen. Die deutsche Bevölkerung weise demnach alle Kennzeichen einer modernen Bevölkerung auf: Junge Menschen unter zwanzig und alte Menschen über sechzig hielten sich die Waage, es gebe seit dreiBig Jahren geburtenschwache Jahrgänge, zudem gebe es ausgebaute Systeme sozialer Sicherung sowie eine große Anziehungskraft für Zuwanderer. Seit den 1960er Jahren ließe sich ein Geburtenrückgang erkennen, der zu einem Sterbefallüberhang führe. Dieser hätte aber bis 2003 durch die Zuwanderung ausgeglichen werden können. Heute liege die durchschnittliche Geburtenzahl pro Frau (Gesamtfruchtbarkeit) bei 1,3. Das Niveau, das die Stärke der Elterngeneration sichern würde, liege aber bei 2,1. In Deutschland gebe es demnach einen stufenweisen Bevölkerungsrückgang. Ein Vergleich mit den anderen europäischen Ländern zeige, dass es in den mittel-, süd- und osteuropäischen Ländern eine ähnliche Situation gebe. Dabei ließe sich Europa unter familienpolitischen und demografischen Gesichtspunkten in vier Räume einteilen: Skandinavien, West-, Süd- und Osteuropa.

Nach Schmid weise Deutschland zudem das Phänomen des demografischen Alterns auf, das sich aus zwei Komponenten zusammensetze: Der Verringerung des Jugendsockels, der Alterung von unten, sowie der steigenden Lebenserwartung, der Alterung von oben. So liege das Durchschnittsalter in Deutschland bei 41 Jahren, während es etwa in Indien bei 20-25 Jahren liege. Bei seiner Vorausberechnung wies Schmid darauf hin, dass bis 2050 ein Drittel des Erwerbspotentials (35-50 Jahre) wegbrechen werde. Alle kompensatorischen Maßnahmen stünden deshalb immer unter dem Diktat des Jugendschwundes. Beide Aspekte hätten schwerwiegende soziale und ökonomische Konsequenzen, die ein neues Denken in Deutschland notwendig machten. Dazu könne jeder Einzelne seinen Beitrag leisten, da jeder seinen Anteil an dieser demografischen Entwicklung habe.

\section{Fazit}

Im Rahmen dieser Tagung wurde die Rolle Deutschlands in der Europäischen Union aus politischer, rechtlicher, wirtschaftlicher, sprachlicher und demografischer Perspektive beleuchtet. Sie bot insoweit einen umfassenden Überblick über die vielschichtigen Aspekte und Auswirkungen der Mitgliedschaft Deutschlands in der Europäischen Union und bot Gelegenheit zu einer regen interdisziplinären Diskussion, in der eine Vielzahl von Einzelfragen angesprochen werden konnten. Insgesamt unterstrich diese Tagung, wie tief die Europäische Union mittlerweile in die Politik ihrer Mitgliedstaaten eingreift und dass Europapolitik heutzutage wohl mit gutem Recht als Teil der Innenpolitik bezeichnet werden kann. Die Tagung verdeutlichte aber umgekehrt auch, wie vielschichtig und damit wie unübersichtlich sich der europäische Integrationsprozess mittlerweile gestaltet und wie wichtig der Austausch zwischen den verschiedenen Wissenschaften geworden ist, um ein umfassendes Bild der Rolle Deutschlands in der Europäischen Union zeichnen zu können. 